\title{
Considerations for patient selection: Prepectoral versus subpectoral implant-based breast reconstruction
}

\author{
Jun Young Yang ${ }^{1}$, Chan Woo Kim ${ }^{1}$, Jang Won Lee ${ }^{1}$, Seung Ki Kim² ${ }^{2}$ Seung Ah Lee ${ }^{2}$, Euna Hwang ${ }^{1}$ \\ Departments of ${ }^{1}$ Plastic and Reconstructive Surgery and ${ }^{2}$ General Surgery, CHA Bundang Medical Center, CHA University School of \\ Medicine, Seongnam, Korea
}

Background In recent years, breast implants have been frequently placed in the subcutaneous pocket, in the so-called prepectoral approach. We report our technique of prepectoral implant-based breast reconstruction (IBR), as well as its surgical and aesthetic outcomes, in comparison with subpectoral IBR. We also discuss relevant considerations and pitfalls in prepectoral IBR and suggest an algorithm for the selection of patients for IBR based on our experiences.

Methods We performed 79 immediate breast reconstructions with a breast implant and an acellular dermal matrix (ADM) sling, of which 47 were subpectoral IBRs and 32 were prepectoral IBRs. Two-stage IBR was performed in 36 cases (20 subpectoral, 16 prepectoral), and direct-to-implant IBR in 43 cases (27 prepectoral, 16 subpectoral). The ADM sling supplemented the inferolateral side of the breast prosthesis in the subpectoral group and covered the entire anterior surface of the breast prosthesis in the prepectoral group.

Results The postoperative pain score was much lower in the prepectoral group than in the subpectoral group (1.78 vs. 7.17). The incidence of seroma was higher in the prepectoral group (31.3\% vs. $6.4 \%)$. Other postoperative complications, such as surgical site infection, flap necrosis, implant failure, and wound dehiscence, occurred at similar rates in both groups. Animation deformities developed in $8.5 \%$ of patients in the subpectoral group and rippling deformities were more common in the prepectoral group (21.9\% vs. $12.8 \%$ ).

Conclusions The indications for prepectoral IBR include moderately-sized breasts with a thick well-vascularized mastectomy flap and concomitant bilateral breast reconstruction with prophylactic mastectomy.

Keywords Breast neoplasms / Breast implant / Mammaplasty
Correspondence: Euna Hwang Department of Plastic and Reconstructive Surgery, CHA Bundang Medical Center, CHA University School of Medicine, 59 Yatap-ro, Bundang-gu, Seongnam 13496, Korea Tel: +82-31-780-5280 Fax: +82-31-780-5285 E-mail: pshwang70@gmail.com

This article was presented at the PRS Korea 2018 on November 9-11, 2018, on Seoul, Korea.

The authors would like to thank Dr. Chang-Ok Seo, a professor in the Department of Radiation Oncology, Bundang CHA Medical Center, CHA University, Seongnam, South Korea for assistance with this article.

Received: March 29, $2019 \bullet$ Revised: October 13, $2019 \bullet$ Accepted: October 22, 2019

pISSN: 2234-6163 • elSSN: 2234-6171 • https://doi.org/10.5999/aps.2019.00353・Arch Plast Surg 2019;46:550-557

\section{INTRODUCTION}

Breast reconstruction methods are generally categorized as those that use autologous tissue, those that use a commercially available breast prosthesis, or a combination of both. In prosthesis-based breast reconstruction, an implant is usually placed under total muscle coverage or a dual plane that is created with the pectoralis major muscle, either with or without acellular dermal 
matrix (ADM). In recent years, breast implants have been frequently placed in the subcutaneous pocket, in the so-called prepectoral approach. In the early stages of breast reconstruction, implant-based breast reconstruction (IBR) was routinely performed in the subcutaneous plane in patients who underwent radical mastectomy in which the pectoralis major muscle was sacrificed, but the popularity of this technique has decreased because of a high incidence of capsular contracture and a high rate of implant extrusion [1-3]. Thereafter, modified radical mastectomy preserving the pectoralis major muscle has been frequently performed in patients with breast cancer; in these procedures, the breast implant is placed under the pectoralis major muscle and/or the serratus muscle to protect it [4]. Breasts reconstructed with implants positioned with total muscle coverage frequently show an unnatural appearance and considerable asymmetry compared to the contralateral normal breast, which has a tear-drop appearance.

$\mathrm{ADM}$, a recently developed product, has been used to provide supplemental support for the inferolateral portion of the implant when the pectoralis major muscle is lacking and to correct rippling deformities in IBR since 2001 [5]. The so-called dualplane breast reconstruction technique, in which the superior part of the breast implant is covered with the pectoralis major muscle, while the inferior part is covered with an $\mathrm{ADM}$ sling, has gained popularity $[6,7]$. Subcutaneous IBR has been reattempted with the assistance of ADM. In 2017, Woo et al. [8] reported a series of subcutaneous IBR procedures, wherein a $16 \times 20 \mathrm{~cm}$ ADM sheet was used to cover the entire anterior surface of the breast implant placed in front of the pectoralis major muscle in patients who underwent nipple-sparing mastectomy. Since then, prepectoral IBRs have been attempted and some initial case series have been reported $[9,10]$.

Herein, we report our technique of prepectoral IBR, along with its surgical and aesthetic outcomes, in comparison with cases of subpectoral IBR. We also discuss the considerations and pitfalls of prepectoral IBR and suggest an algorithm for the selection of patients for IBR based on our experiences.

\section{METHODS}

Between March 2017 and September 2018, we performed a total of 79 immediate breast reconstructions using a breast implant (Mentor CPG silicone gel implant; Johnson \& Johnson, New Brunswick, NJ, USA) and an ADM sling (47 subpectoral, 32 prepectoral). Through a retrospective chart review, the demographic information, clinical data, and surgical outcomes of patients who underwent IBR were obtained. This study was approved and performed under CHA Bundang Medical Center
Institutional Review Board guidelines (IRB No. 2018-11-031004).

The factors influencing the choice of the prepectoral plane were patient consent, tumors located more than $1 \mathrm{~cm}$ from the anterior surface of the pectoralis muscle or chest wall, and a clinically well-perfused mastectomy flap without visible dermis. All reconstructions were performed by a single surgeon $(\mathrm{EH})$, while the mastectomies were performed by two different breast surgeons (S.K.K. and S.A.L.).

In total, 36 two-stage IBR procedures (20 subpectoral and 16 prepectoral) and 43 direct-to-implant (DTI) IBR procedures (27 prepectoral and 16 subpectoral) were performed. An ADM sling supplemented the inferolateral side of the breast prosthesis in the subpectoral group and covered the entire anterior surface of the breast prosthesis in the prepectoral group. Two kinds of ADM (MegaDERM; L\&C Bio, Seongnam, Korea and CG CRYODERM; CG Bio, Seongnam, Korea) were used in all patients.

\section{Operative technique of prepectoral IBR}

The oncologic breast surgery team performed total mastectomy procedures (either nipple-sparing or sacrificing the nipple-areolar complex) in this series. In the first step of prepectoral reconstruction, we placed the selected temporary breast sizer in the subcutaneous pocket and temporarily sutured the skin. With the patient in the upright sitting position, the inframammary fold was marked at the same level of the contralateral breast. A large one-piece ADM mesh $(18 \times 14 \mathrm{~cm}$ or $16 \times 14 \mathrm{~cm})$ with a 2.5- to 3.0-mm thickness was draped over the breast mound created with an adequate breast sizer. The inframammary fold level was marked on the ADM mesh $1.5-2 \mathrm{~cm}$ above the lower margin of the $\mathrm{ADM}$. The $\mathrm{ADM}$ mesh was pulled up to cover the entire breast implant and the superior part beyond the superior border of the breast implant. The remaining ADM mesh was cut out and added to the inferolateral side of the ADM mesh to elongate it to completely cover the inferolateral breast implant. After removal of the temporary breast sizer, the ADM was fixed along the inframammary fold with 5-6 pillow anchoring sutures, and the 1.5- to 2-cm-long cuff of the ADM mesh was manipulated into an internally-folded tongue-in-groove pattern and fixed to the underlying deep fascia with \#3-0 polydioxanone (PDS) sutures to support it along the inframammary fold. A permanent breast implant or tissue expander was placed under the ADM mesh, which was pulled up superiorly to cover the breast implant. The superior part of the ADM (usually 2-3 cm from the superior margin of the breast implant; with a triangular shape) was tightly fixed to the pectoralis muscle with \#2-0 Vicryl sutures and/or \#3-0 PDS sutures. The lateral and medial 


\section{Fig. 1. Surgical technique of prepectoral implant-based breast reconstruction}

(A) Acellular dermal matrix (ADM) for prepectoral breast reconstruction. A $14 \times 16 \mathrm{~cm}$ or $14 \times 18 \mathrm{~cm}$ piece of ADM with a $2.5-$ to $3-\mathrm{mm}$ thickness. (B) The superolateral side of the ADM was cut out and added to the inferolateral side of the ADM to elongate it to completely cover the inferolateral side of the breast implant. A 1- to 2-cm-long cuff of the ADM was folded in a groove pattern to support the breast implant along the inframammary fold. (C) Anterior view of the prepectoral reconstruction. The implant was positioned in the prepectoral space and covered with an ADM mesh. (D) Lateral view of the prepectoral reconstruction. The inferior and superior margins of the ADM were fixed to the underlying deep fascia.

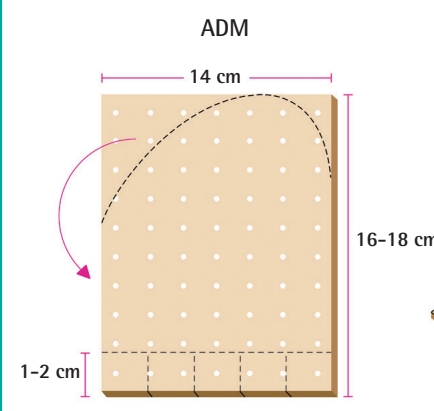

(A)

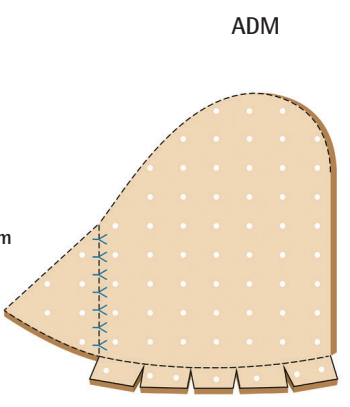

(B)

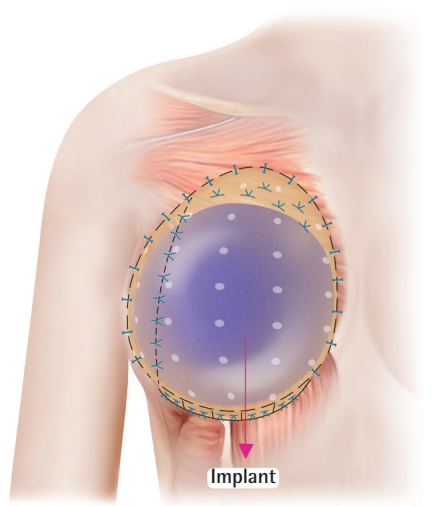

Front view

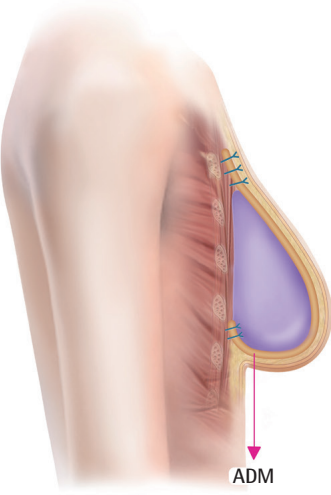

Lateral view (D) margins of the $\mathrm{ADM}$ mesh were also fixed to the underlying muscle and fascia to prevent implant migration or rotation (Fig. 1). Two Jackson-Pratt drains were placed in the lateral and inferior sides and the subcutaneous tissue was repaired with \#3-0 Vicryl, \#4-0 Vicryl, and \#5-0 Nylon sutures. After the inframammary fold was checked again, a compressive dressing was placed with an elastic bandage. The drain was removed once $<30 \mathrm{~mL} /$ day of fluid was collected for 2 consecutive days.

\section{Assessment of postoperative complications and aesthetic outcomes}

Acute postoperative complications were defined as those occurring up to 90 days after reconstructive surgery, including infection, seroma, hematoma, flap necrosis, implant failure, and wound dehiscence. Flap necrosis was defined as a necrotic area over $2 \times 2 \mathrm{~cm}^{2}$, and delayed wound healing referred to wound problems requiring revision due to wound dehiscence.

Outcomes relating to cosmesis, including capsular contracture, rippling deformity, and animation deformity, were assessed by two physicians using chart reviews and photographs obtained at least 3 months after DTI breast reconstruction or implant change in the second stage of IBR. Postoperative pain scores were recorded using the mean numeric rating scale (NRS) value obtained on 2 consecutive days starting on the first postoperative day. Postoperative pain was controlled using patient-controlled analgesia (PCA), which contained $150 \mu \mathrm{g}$ of Sufental (sufentanil citrate, 3 ampoules), and $0.3 \mathrm{mg}$ of Nasea (ramosetron $\mathrm{HCl}, 1$ ampoule) in normal saline (total volume of 100
$\mathrm{mL}$ ) in most patients, or $2 \mathrm{~g}$ of Denogan (propacetamol hydrochloride) three times a day for pain control in patients who complained of side effects of PCA, such as nausea and dizziness. Animation deformity was evaluated in the slightly bent position with patients' palms pressed together strongly. Capsular contracture was estimated using Baker classification system and rippling deformity was evaluated using a grading system suggested by Pantelides and Srinivasan [11] (grade 1, mild: rippling is palpable but not visible; grade 2, moderate: rippling is visible only when a patient bends forward; and grade 3 , severe: rippling is visible in upright standing position).

\section{Statistical analysis}

Statistical analyses were performed using SPSS for Windows version 25.0 (IBM Corp., Armonk, NY, USA). The results are presented as mean \pm standard deviation or as the number of cases with percentage in brackets. For intergroup comparisons, the data were analyzed using the Student t-test, Pearson chi-square test, and Fisher exact test. For all tests, P-values $<0.05$ were considered to indicate statistical significance.

\section{RESULTS}

A total of 79 cases (47 subpectoral, 32 prepectoral) were analyzed in 73 patients. There were 43 DTI reconstruction cases and 36 cases of two-stage IBR with a tissue expander. Six patients underwent bilateral reconstruction, while the remaining 67 patients underwent unilateral reconstruction. The patients' 
Table 1. Patients' demographics in the subpectoral and prepectoral IBR groups

\begin{tabular}{|c|c|c|c|}
\hline Variable & $\begin{array}{l}\text { Subpectoral } \\
\text { reconstruction }\end{array}$ & $\begin{array}{l}\text { Prepectoral } \\
\text { reconstruction }\end{array}$ & P-value \\
\hline No. of case & 47 & 32 & \\
\hline \multicolumn{4}{|l|}{ Patient data } \\
\hline Age (yr) & $46.40 \pm 6.96$ & $48.91 \pm 8.51$ & 0.173 \\
\hline $\mathrm{BMI}\left(\mathrm{kg} / \mathrm{m}^{2}\right)$ & $21.25 \pm 2.86$ & $23.49 \pm 3.71$ & 0.003 \\
\hline Smoking history & $8(17.0)$ & $4(12.5)$ & 0.434 \\
\hline Active smoker & $3(6.4)$ & $3(9.4)$ & 0.894 \\
\hline Ex-smoker & $5(10.6)$ & $1(3.1)$ & 0.216 \\
\hline Diabetes & $2(4.3)$ & $3(9.4)$ & 0.359 \\
\hline Two staged IBR & $20(42.6)$ & $16(50.0)$ & 0.514 \\
\hline Direct to Implant & $27(57.4)$ & $16(50.0)$ & 0.514 \\
\hline Median follow-up (wk) & 55.9 & 47.7 & \\
\hline \multicolumn{4}{|l|}{ Histology } \\
\hline Invasive tumor & $33(70.2)$ & $24(75.0)$ & 0.641 \\
\hline DCIS \& LCIS & $12(25.5)$ & $7(21.9)$ & 0.709 \\
\hline No tumor (prophylaxis) & $2(4.3)$ & $1(3.1)$ & 0.796 \\
\hline \multicolumn{4}{|l|}{ Axillary node operation } \\
\hline Nodal clearance & $8(17.0)$ & $1(3.1)$ & 0.056 \\
\hline Sentinel node biopsy & $35(74.5)$ & $30(93.8)$ & 0.028 \\
\hline No axillary surgery & $4(8.5)$ & $1(3.1)$ & 0.334 \\
\hline \multicolumn{4}{|l|}{ Miscellaneous information } \\
\hline Chemotherapy & $22(46.8)$ & $14(43.8)$ & 0.789 \\
\hline Adjuvant radiotherapy & $7(14.9)$ & $2(6.3)$ & 0.235 \\
\hline Specimen weight (g) & $272.03 \pm 135.22$ & $343.88 \pm 138.31$ & 0.020 \\
\hline Implant volume (mL) & $284.07 \pm 84.22$ & $290.94 \pm 85.29$ & 0.798 \\
\hline TE initial inflation volume (mL) & $167.00 \pm 90.97$ & $213.57 \pm 115.47$ & 0.187 \\
\hline \multicolumn{4}{|c|}{$\begin{array}{l}\text { Values are presented as mean } \pm \text { SD or number (\%). } \\
\text { BMI, body mass index; IBR, implant-based breast reconstruction; DCIS, ductal } \\
\text { carcinoma in-situ; LCIS, lobular carcinoma in-situ; TE, tissue expander. } \\
\text { P-values area calculated by Student t-test, Pearson chi-square test and Fisher } \\
\text { exact test. }\end{array}$} \\
\hline
\end{tabular}

characteristics are shown in Table 1. The patients' mean age was 46.40 years in the subpectoral group and 48.91 years in the prepectoral group. The mean breast tissue weight after mastectomy was $272.03 \mathrm{~g}$ in the subpectoral group and $343.88 \mathrm{~g}$ in the prepectoral group. The patients' demographics were similar between the two groups (Table 1). The median follow-up period was 50.0 weeks in the entire population, 47.7 weeks in the prepectoral group, and 55.9 weeks in the subpectoral group.

On average, drains remained present for 11.06 days in the subpectoral group vs. 8.48 days in the prepectoral group, while the postoperative pain score was 7.17 in the subpectoral group vs. 1.78 in the prepectoral group. The patients who underwent prepectoral reconstruction complained of much less pain on the first and second postoperative days than those who underwent subpectoral reconstruction. Seroma was more common in the prepectoral group (6.4\% in the subpectoral group vs. $31.3 \%$ in the prepectoral group). In patients who underwent prepectoral reconstruction, seromas most commonly occurred in the upper pole of the breast implant. Hematoma occurred in no cases in
Table 2. Comparison of outcomes between prepectoral and subpectoral IBR

\begin{tabular}{|c|c|c|c|c|}
\hline Variable & $\begin{array}{l}\text { Subpectoral } \\
\text { reconstruction }\end{array}$ & $\begin{array}{l}\text { Prepectoral } \\
\text { reconstruction }\end{array}$ & P-value & $\begin{array}{l}\text { Odds } \\
\text { ratio }\end{array}$ \\
\hline No. of case & 47 & 32 & & \\
\hline Drain & & & & - \\
\hline $\begin{array}{l}\text { Drain maintain day } \\
\text { (day) }\end{array}$ & $11.06 \pm 5.33$ & $8.48 \pm 2.72$ & 0.009 & \\
\hline $\begin{array}{l}\text { Total drain volume } \\
(\mathrm{mL})\end{array}$ & $866 \pm 674$ & $745 \pm 622$ & 0.419 & \\
\hline $\begin{array}{l}\text { Pain score } \\
\text { NRS (0-10) }\end{array}$ & $7.17 \pm 1.44$ & $1.78( \pm 1.79)$ & $<0.001$ & - \\
\hline $\begin{array}{l}\text { Postoperative } \\
\text { complications } \\
\text { ( } \leq 90 \text { day) }\end{array}$ & & & & \\
\hline Infection & $1(2.1)$ & $1(3.1)$ & 0.782 & - \\
\hline Seroma & $3(6.4)$ & $10(31.3)$ & 0.003 & 6.67 \\
\hline Hematoma & $7(14.9)$ & 0 & 0.038 & 0 \\
\hline Flap necrosis & $6(12.8)$ & $4(12.5)$ & 0.972 & - \\
\hline Implant failure & $1(2.1)$ & $1(3.1)$ & 0.782 & - \\
\hline $\begin{array}{l}\text { Delayed wound } \\
\text { healing }\end{array}$ & $15(31.9)$ & $8(25.0)$ & 0.507 & - \\
\hline $\begin{array}{l}\text { Postoperative } \\
\text { complications } \\
\text { (>90 day) }\end{array}$ & & & & - \\
\hline Latent seroma & $1(2.1)$ & $2(6.3)$ & 0.347 & \\
\hline Infection & 0 & $1(3.1)$ & 0.223 & \\
\hline Implant failure & 0 & 0 & - & \\
\hline Aesthetic outcomes & & & & - \\
\hline Rippling & $6(12.8)$ & $7(21.9)$ & 0.284 & \\
\hline Grade I & 0 & $1(3.1)$ & 0.226 & \\
\hline Grade lla & $1(2.1)$ & $2(6.3)$ & 0.785 & \\
\hline Grade Illb & $3(6.4)$ & $2(6.3)$ & 0.636 & \\
\hline Grade III & $2(4.3)$ & $2(6.3)$ & 0.699 & \\
\hline Animation deformity & $4(8.5)$ & 0 & 0.090 & \\
\hline Capsular contracture ${ }^{a}$ & $1(2.1)$ & 0 & 0.406 & \\
\hline \multicolumn{5}{|c|}{$\begin{array}{l}\text { Values are presented as mean } \pm \mathrm{SD} \text { or number (\%). } \\
\text { IBR, implant-based breast reconstruction; NRS, numeric rating scale. } \\
\text { P-values area calculated by Student t-test, Pearson chi-square test and Fisher } \\
\text { exact test. Statistically meaningful odds ratio value is described. } \\
\text { a'Baker's grade III. }\end{array}$} \\
\hline
\end{tabular}

the prepectoral group, but occasionally developed in the subpectoral group ( $0 \%$ vs. $14.9 \%$, respectively). Other postoperative complications, such as surgical site infection, flap necrosis, implant failure, and wound dehiscence, occurred with a similar frequency in both groups (Table 2). A delayed infection occurred in one patient in the prepectoral group.

In the analysis of aesthetic outcomes, animation deformities were observed in $8.5 \%$ of cases in the subpectoral group and Baker grade III capsular contracture occurred in one case in the subpectoral group. Rippling deformities were more frequently observed in the prepectoral group (12.8\% in the subpectoral group vs. $21.9 \%$ in the prepectoral group) (Table 2). Severe rippling deformities requiring correction occurred in some patients in the prepectoral group who had large ptotic breasts. We per- 
formed four revisional procedures for rippling deformities using fat grafts (three cases) or filler injections (one case).

\section{DISCUSSION}

Prepectoral approaches have been attempted with the assistance of ADM. A variety of studies have reported the benefits and adverse effects of $A D M$ slings in IBR. The advantages of $A D M$ include a reduced incidence of capsular contracture, and ADM tends to protect the skin from the adverse effects of radiotherapy $[12,13]$. It also enables the inframammary fold to be adjusted to a proper position [14]. Breast implant rippling and visibility can be reduced by covering the inferolateral part of the implant [15]. These advantages of ADM slings seem to overcome their high cost and adverse effects, including an increased seroma rate. In recent years, prepectoral breast reconstruction (subcutaneous breast reconstruction with an $\mathrm{ADM}$ sling covering the entire breast implant placed in the original space of the breast) has been receiving increased attraction. Prepectoral placement of the breast implant eliminates the undermining and detachment of the pectoralis muscle from the chest wall, with several advantages, including a considerable reduction in postoperative pain, the absence of animation deformities, and less discomfort during movement of the arm and shoulder. However, concerns persist about its application in patients with thin mastectomy flaps, insufficiently vascularized flaps, or postoperative rippling deformities.

Several reports have compared the prepectoral and subpectoral approaches. According to Nahabedian and Cocilovo [16], surgical site infections occurred more frequently in prepectoral reconstructions ( $8.1 \%$ vs. $4.8 \%)$, and seroma formation and postoperative hematoma were likewise more common in patients who underwent prepectoral reconstructions (seroma: $4.8 \%$ vs. $2.4 \%$ and hematoma: $4.8 \%$ vs. $0 \%$ ). Chandarana et al. [17] concluded that there were no specific differences in acute complications [17]. Baker et al. [18] reported that there was no significant difference in length of stay or early morbidities, but that patients who received prepectoral reconstructions were dissatisfied with the visible rippling of the implant (7 of 13 in the prepectoral group vs. 2 of 17 in the subpectoral group).

In our series, rippling deformities developed in seven of 32 cases in the prepectoral group (21.9\%). There were two cases of grade III severe rippling deformities and four cases of grade II moderate rippling deformities. The incidence of rippling deformities was lower than was presented in a previous report [18]. We think that this discrepancy can be explained by our use of thick $\mathrm{ADM}$ and the fact that we usually performed prepectoral IBR in patients with a thick skin flap after total mastectomy in which the thin periareolar skin was sacrificed. In addition, Asian women generally have moderate-sized or small breasts without severe ptosis. Two of our patients with severe rippling deformities had large breasts with moderate ptosis and underwent nipple-sparing mastectomy and prepectoral IBR. Mastectomy of a large ptotic breast produces a large area of thin skin, which is wider than the area needed to cover an adequately sized breast implant. The left-over thin skin flap is likely to result in a rippling deformity. Methods of correcting rippling deformities include fat grafting on the subcutaneous tissue to decrease the degree of rippling, use of a larger breast implant than the patient's actual breast volume, or reduction of the breast pocket by capsulorrhaphy with or without reduction of the excessive skin. In bilateral cases, rippling deformities are rare, because a breast implant larger than the previous breast volume is used. The rate of wound problems or flap necrosis was similar in both groups. The rate of flap necrosis was relatively high in both groups, because we did not remove poorly vascularized areas of the mastectomy flap intraoperatively. We repaired the necrotic areas of mastectomy skin flaps after the area was fully demarcated.

We performed prepectoral IBR in all patients except those with a tumor close to the pectoralis muscle or a very thin mastectomy skin flap. After performing 32 prepectoral IBRs, we needed to establish reasonable selection criteria for prepectoral IBR to ensure better outcomes. To obtain good IBR outcomes, the most important consideration is patient selection with the goal of minimizing postoperative complications. We created an algorithm for patient selection based on our experiences (Fig. 2). First, before the operation, breast magnetic resonance imaging (MRI) should be reviewed to assess the tumor location and size. If the tumor is located close to the chest wall, especially the pectoralis major muscle, or if the tumor is $>3 \mathrm{~cm}$, subpectoral IBR is preferable. It is easy to detect cancer recurrence when the pectoralis major muscle is anteriorly displaced in front of a breast implant, rather than behind the prosthesis. Furthermore, in cases of postmastectomy radiation therapy (PMRT), the radiation field can be decreased and limited to the superficial layer above the breast implant. Most of our cases were early-stage breast cancers. Therefore, very few patients underwent PMRT and the rate of radiation therapy-induced pathologic capsular contracture was relatively low. The reason for this might be that PMRT at our institution was conducted in accordance with the European Society of Radiation and Oncology consensus guideline, which recommends the anterior surface of the pectoralis major muscle as the dorsal border of the chest wall clinical target volume (CTV), stating that "unless invasion was demonstrated (tumor stage T4a and T4c) there is no reason for routinely including the major pectoral muscle and the ribs in CTV_thorac- 
Fig. 2. Implant-based breast reconstruction selection algorithm

MRI, magnetic resonance imaging; TE, tissue expander; NSM, nipple-sparing mastectomy; SSM, skin-sparing mastectomy; TM, total mastectomy.

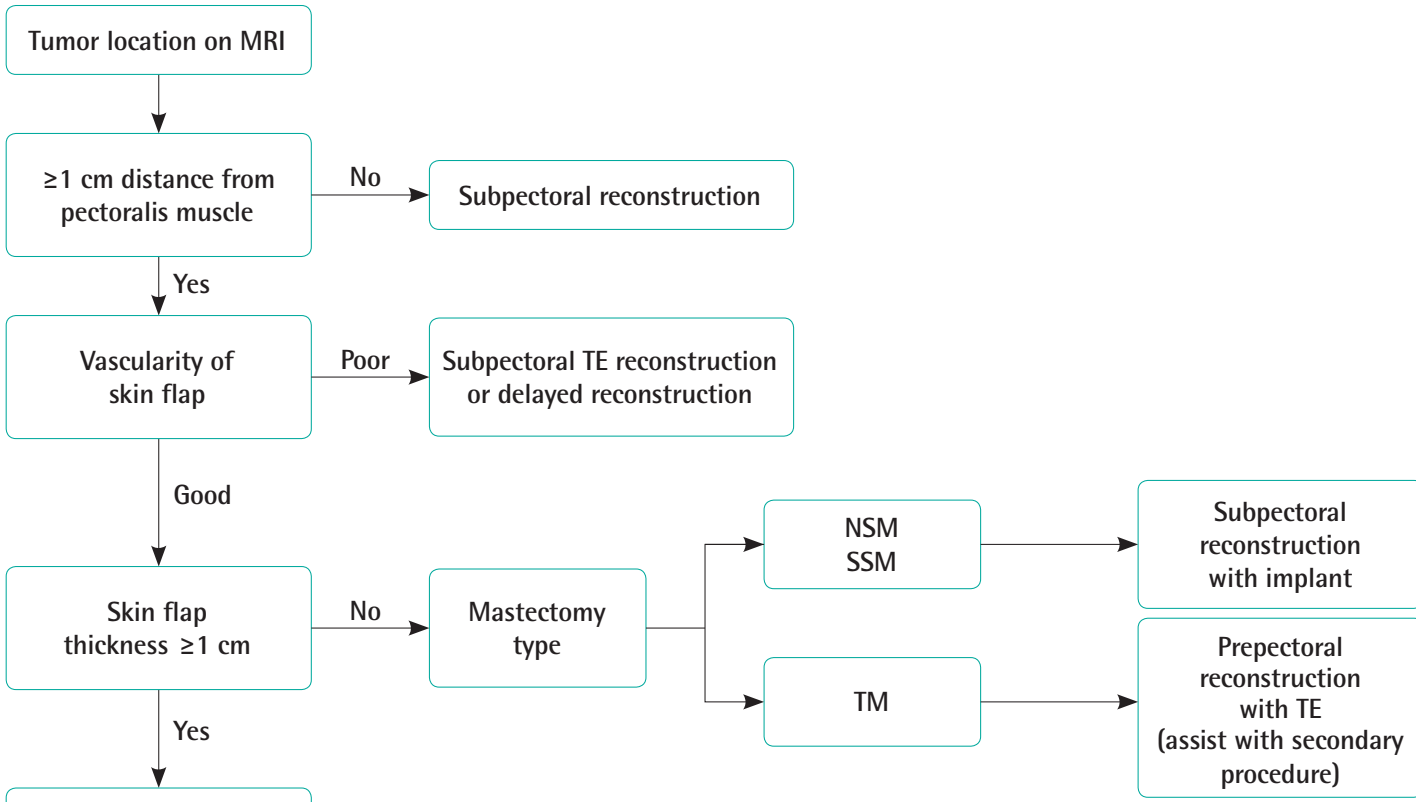

Prepectoral reconstruction with TE of implant

ic wall" [19]. Furthermore, according to the 2019 ESTRO (European Society for Radiotherapy and Oncology) guideline, if the dorsal fascia of the breast does not show cancer involvement, the CTVp chest wall (where "p" indicates primary) for PMRT should not include the deep lymphatic plexus, and the dorsal margin of the CTV should be at the ventral side of the implant in IBR using a retro-pectoral implant. After IBR using a prepectoral positioned implant, the CTVp_chest wall is composed of two parts, reflecting the division of the prepectoral volume into two parts by the implant (the ventral part between the skin and the implant, containing the subcutaneous lymphatic plexus and eventual residual glandular tissue, and the dorsal part between the implant and the pectoral muscle/chest wall, containing eventual residual glandular tissue), which should only be included in the presence of adverse tumor-related factors [20].

We set the safety margin at a distance of $1 \mathrm{~cm}$ from the pectoralis major muscle when the prepectoral approach was selected. In some studies $[9,10]$, a 0.5 -cm distance was suggested, but we think that $0.5 \mathrm{~cm}$ is too narrow because breast MRI is usually taken in the prone position and the breast tissue is shown as more elongated than its actual position. Therefore, we prefer a $1.0-\mathrm{cm}$ distance from the anterior surface of the pectoralis muscle on MRI to secure a substantial safety margin. When the cancer is located more than $1 \mathrm{~cm}$ from the pectoralis major muscle on MRI, prepectoral IBR can be preoperatively considered. Then, we assess the vascularity of the mastectomy flap in the operative field after the mastectomy is performed by the breast surgery team. If the vascularity of the skin flap is estimated to be poor in a clinical examination (confirming the subdermal plexus of the skin flap) or based on fluorescence angiography with indocyanine green, delayed reconstruction or subpectoral IBR with a tissue expander is considered depending on the extent of the poorly vascularized area. The next step is measurement of the thickness of the mastectomy flap. If the thickness approaches $1 \mathrm{~cm}$ or the subcutaneous fat tissue is well-preserved in the mastectomy skin flap, the flap is considered to be well-vascularized and a very good candidate for prepectoral IBR. However, skin flaps with a thickness approaching $1 \mathrm{~cm}$ are uncommon, especially in patients with large ptotic breasts or in slim patients, whose skin flaps are relatively thin. Even if the mastectomy skin flap is less than $1 \mathrm{~cm}$ thick, skin flap vascularity is not problematic if the subdermal plexus traveling between the subcutaneous fat globules are not broken down during mastectomy. If the mastectomy flap is too thin and its vascularity is undetermined, we prefer subpectoral IBR. For mastectomy flaps with good vascularity, but insufficient subcutaneous fat tissue, subpectoral IBR is also preferable. A thin mastectomy flap is likely to develop a rippling deformity. In addition, large ptotic breasts are not 
good candidates for prepectoral IBR, because they usually have thin skin. Thin skin envelopes are loose and do not usually fit the breast implant counterpart of the contralateral normal breast, and especially in cases of nipple- or skin-sparing mastectomy, rippling deformities develop frequently. However, in patients who undergo breast reconstruction using a tissue expander following total mastectomy sacrificing the nipple-areolar complex, the mastectomy skin flap is rather thick and secondary procedures such as fat grafting, capsulorrhaphy, or other ancillary procedures that correct the rippling deformity can be performed in the second stage. Prepectoral IBR can be also used in those patients.

We think that rippling deformities in patients who undergo IBR are influenced by various factors, including mastectomy flap thickness, breast implant type and size, ADM thickness and fixation technique, mastectomy type (nipple- or skin-sparing mastectomy, total mastectomy), and reconstruction method (DTI or tissue expander). Favorable conditions for preventing rippling deformity in prepectoral IBR include a thick mastectomy flap with sufficient subcutaneous fat tissue, moderate to high body mass index, small to moderately-sized breasts, lack of ptosis, a tight skin envelope, use of an oversized breast implant, and tight fixation of a thick ADM mesh.

To conclude, favorable indications for prepectoral IBR include moderately-sized breasts with a thick well-vascularized mastectomy flap and concomitant bilateral breast reconstruction including prophylactic mastectomy. Prepectoral IBR may be a good surgical option in properly selected cases.

\section{NOTES}

\section{Conflict of interest}

No potential conflict of interest relevant to this article was reported.

\section{Ethical approval}

The study was approved by the Institutional Review Board of CHA Bundang Medical Center (IRB No. 2018-11-031-004) and performed in accordance with the principles of the Declaration of Helsinki.

\section{Author contribution}

Conceptualization: Hwang E. Data curation: Hwang E. Formal analysis: Kim CW. Methodology: Hwang E. Project administration: Hwang E, Kim SK, Lee SA. Writing - original draft: Yang JY. Visualization: Lee JW. Writing - review \& editing: Hwang E. Approval of final manuscript: all authors.

\section{ORCID}

Jun Young Yang https://orcid.org/0000-0003-4995-9813

Chan Woo Kim https://orcid.org/0000-0002-7441-8480

Jang Won Lee https://orcid.org/0000-0002-3885-5878

Euna Hwang https://orcid.org/0000-0002-0077-3092

\section{REFERENCES}

1. Gruber RP, Kahn RA, Lash H, et al. Breast reconstruction following mastectomy: a comparison of submuscular and subcutaneous techniques. Plast Reconstr Surg 1981;67:312-7.

2. Schlenker JD, Bueno RA, Ricketson G, et al. Loss of silicone implants after subcutaneous mastectomy and reconstruction. Plast Reconstr Surg 1978;62:853-61.

3. Snyderman RK, Guthrie RH. Reconstruction of the female breast following radical mastectomy. Plast Reconstr Surg 1971;47:565-7.

4. Apfelberg DB, Laub DR, Maser MR, et al. Submuscular breast reconstruction: indications and techniques. Ann Plast Surg 1981;7:213-21.

5. Duncan DI. Correction of implant rippling using allograft dermis. Aesthet Surg J 2001;21:81-4.

6. Breuing KH, Warren SM. Immediate bilateral breast reconstruction with implants and inferolateral AlloDerm slings. Ann Plast Surg 2005;55:232-9.

7. Spear SL, Parikh PM, Reisin E, et al. Acellular dermis-assisted breast reconstruction. Aesthetic Plast Surg 2008;32:41825.

8. Woo A, Harless C, Jacobson SR. Revisiting an old place: single-surgeon experience on post-mastectomy subcutaneous implant-based breast reconstruction. Breast J 2017;23:54553.

9. Jones G, Yoo A, King V, et al. Prepectoral immediate directto-implant breast reconstruction with anterior AlloDerm coverage. Plast Reconstr Surg 2017;140(6S):31S-38S .

10. Sbitany H, Piper M, Lentz R. Prepectoral breast reconstruction: a safe alternative to submuscular prosthetic reconstruction following nipple-sparing mastectomy. Plast Reconstr Surg 2017; 140:432-43.

11. Pantelides NM, Srinivasan JR. Rippling following breast augmentation or reconstruction: aetiology, emerging treatment options and a novel classification of severity. Aesthetic Plast Surg 2018;42:980-5.

12. Ganske I, Verma K, Rosen H, et al. Minimizing complications with the use of acellular dermal matrix for immediate implant-based breast reconstruction. Ann Plast Surg 2013; 71:464-70.

13. Salzberg CA, Ashikari AY, Berry C, et al. Acellular dermal 
matrix-assisted direct-to-implant breast reconstruction and capsular contracture: a 13-year experience. Plast Reconstr Surg 2016;138:329-37.

14. Sbitany H, Serletti JM. Acellular dermis-assisted prosthetic breast reconstruction: a systematic and critical review of efficacy and associated morbidity. Plast Reconstr Surg 2011; 128:1162-9.

15. Lohmander F, Lagergren J, Roy PG, et al. Implant based breast reconstruction with acellular dermal matrix: safety data from an open-label, multicenter, randomized, controlled trial in the setting of breast cancer treatment. Ann Surg 2019;269:836-41.

16. Nahabedian MY, Cocilovo C. Two-stage prosthetic breast reconstruction: a comparison between prepectoral and partial subpectoral techniques. Plast Reconstr Surg 2017;140(6S): 22S-30S.

17. Chandarana MN, Jafferbhoy S, Marla S, et al. Acellular der- mal matrix in implant-based immediate breast reconstructions: a comparison of prepectoral and subpectoral approach. Gland Surg 2018;7(Suppl 1):S64-9.

18. Baker BG, Irri R, MacCallum V, et al. A prospective comparison of short-term outcomes of subpectoral and prepectoral Strattice-based immediate breast reconstruction. Plast Reconstr Surg 2018;141:1077-84.

19. Offersen BV, Boersma LJ, Kirkove C, et al. ESTRO consensus guideline on target volume delineation for elective radiation therapy of early stage breast cancer. Radiother Oncol 2015;114:3-10.

20. Kaidar-Person O, Vrou Offersen B, Hol S, et al. ESTRO ACROP consensus guideline for target volume delineation in the setting of postmastectomy radiation therapy after implant-based immediate reconstruction for early stage breast cancer. Radiother Oncol 2019;137:159-66. 